\title{
HIV and ZIKA in Pregnancy: parallel stories and new challenges
}

\begin{abstract}
On February 1, 2016, the World Health Organization (WHO) declared the Zika virus outbreak a Public Health Emergency of International Concern (PHEIC). Because Zika virus is a relatively new infection with several transmission routes that include mosquito bites, sexual and possibly blood-related, we present the case for a parallel between the Zika epidemic and the AIDS epidemic for issues that relate particularly to pregnancy and epidemic response. We will discuss the many similarities between both epidemics while acknowledging that the viruses are different and the pathophysiology and disease manifestation are also different. What can we learn of the three decades of awareness campaigns, community involvement, clinical, behavior and prevention research, activism and policy-making related to HIV/AIDS? How can the lessons and experience of dealing with the HIV epidemic can help us deal with the ongoing Zika epidemic? Is there a roadmap that we can follow? Our position is that we can and we should.
\end{abstract}

Parallels between the Zika and HIV/AIDS epidemics particularly in relation to pregnancy can be established. Lessons and successes from the HIV/AIDS epidemic control efforts can guide us towards comprehensive approaches to improve the health of women and infants at risk for Zika.
Volume 5 Issue 6 - 2016

\author{
Carmen D Zorrilla,' Ana Marרa Mosquera, ${ }^{3}$ \\ Silvia Rabionet, ${ }^{2}$ Juana Rivera-Vinas' \\ 'Obstetrics and Gynecology Department, UPR School of \\ Medicine, Puerto Rico \\ 2 Nova Southeastern School of Pharmacy, Puerto Rico \\ ${ }^{3}$ Obstetrics and Gynecology Department, UPR School of \\ Medicine, Puerto Rico
}

\begin{abstract}
Correspondence: Carmen D Zorrilla, Obstetrics and Gynecology Department, UPR School of Medicine, UPR School of Medicine, Maternal-Infant Studies Center (CEMI), PO Box. 365067 San Juan, Puerto Rico, 00936-5067,

Email carem.zorrilla@upr.edu
\end{abstract}

Received: November 27, 2016 | Published: December 15, 2016

\section{Keywords: HIV, ZIKA, Pregnancy}

\section{Introduction}

On February 1, 2016, the World Health Organization (WHO) declared the Zika virus outbreak a Public Health Emergency of International Concern (PHEIC). The WHO Secretariat briefed the Committee on the clusters of microcephaly and Guillain-Barré Syndrome (GBS) that have been temporally associated with Zika virus transmission in some settings. ${ }^{1}$

Because Zika virus is a relatively new infection with several transmission routes that include mosquito bites, sexual and possibly blood-related, we present the case for a parallel between the Zika epidemic and the AIDS epidemic for issues that relate particularly to pregnancy and epidemic response. For Obstetricians who provided care to pregnant women living with HIV during the first decade of the AIDS epidemic, this new Zika epidemic among pregnant women present many similarities. The conversations and counseling we are now having with our pregnant patients in the middle of a Zika epidemic include many common aspects. For example: it is a new disease and there is not much information about how to predict fetal adverse outcomes, there is a need for closer maternal follow-up and the need to provide longitudinal care and evaluations to infants. Options to continue or to interrupt the pregnancy should be available and counseling should be non-judgmental. Prenatal diagnosis of fetal infection is not available for Zika and the testing might be inconclusive. These were the same issues confronted by women living with HIV in the early years of the epidemic. Invasive procedures were discouraged for the fear of facilitating percutaneous infection. There were no treatments, no vaccines, and testing was cumbersome. Results of HIV testing would take about two weeks on most places and longer on places with less infrastructure. Zika testing is not commercially available and local health departments might take longer than two weeks for results. Because of the implications of an HIV diagnosis, testing algorithms required pre and post counseling. This has been eliminated for HIV with the availability of point of care, rapid HIV testing and Home HIV testing. Still Zika presents difficulties for testing and diagnosis and therefore counseling of pregnant women needs to consider those factors. Delays with positive results might affect the options and timing for pregnancy terminations or other decisions during the pregnancy.

We will discuss the many similarities between both epidemics while acknowledging that the viruses are different and the pathophysiology and disease manifestation are also different. Nevertheless the following issues might have some commonalities: Zika (as initially HIV) was a new and unknown viral disease, which can affect fetuses in different ways including an embryopathy (Microcephaly for Zika, AIDS embriopathy for HIV). ${ }^{2}$ Pregnancies can be affected with other adverse outcomes including intrauterine fetal deaths, and the impact on exposed fetuses is unclear. ${ }^{3}$ Counseling of pregnant women with Zika can be difficult due to the many unanswered questions. Both viruses are sexually transmitted, initially with no treatments or vaccines, causing stigma, fear and social isolation, both are/were seen as affecting tourism, with mixed messages in public response for which some include travel restrictions. Furthermore the prevention strategies and messages are focused on individual behavior change.

For those caring for pregnant women and women of reproductive age, both diseases are important to address, counsel and promote all prevention strategies because of the long term impact and implications for infants exposed in utero to any one of these viral agents.

\section{Materials and methods}

While most of the issues we will discuss are based on the authors experiences with both epidemics as providers and researchers, literature searches were performed to include evidence of HIVtargeted prevention interventions, response to the epidemic, some official guidelines and recommendations for the management of 
pregnant women either living with HIV or suspected to be infected or exposed to Zika virus. Interviews with pregnant women with Zika or with HIV during group prenatal care is the source of some testimonies. The women consented to provide socio-demographic and clinical information. The Prenatal care consent includes sections on recordings and photographs. This study was approved by the Medical Sciences Campus IRB (Protocol Number: 1350316).

Recommendations regarding prevention and management of Zika and/or HIV focusing on Pregnancy were reviewed including those by the WHO, ACOG, CDC and the USPHS. ${ }^{4-7}$ Counseling of pregnant women per guidelines was reviewed and some of the experiences of women with Zika and women with HIV were included as testimonies. Public response includes media coverage: New York Times, Wall Street Journal, Rolling Stone magazine, CBS 60 minutes and others was reviewed. ${ }^{8-11}$ Review of Condom use as strategy for HIV prevention. ${ }^{12-14}$

\section{Results and discussion}

As stated earlier, a new viral infection that can cause birth defects and can be transmitted by vectors (mosquitoes) or sexually remind us of the earlier approach and response to the AIDS epidemic. Rather than re-thinking or re-inventing the wheel, we propose to look back at our experience with the AIDS epidemic. There are lessons and mistakes that had to be corrected in order to respond to the epidemic while respecting human rights, and patients' autonomy. The initial response of many countries to the AIDS epidemic in trying to avoid publicity in order to preserve income from tourism has been seen again with the Zika epidemic. The public conversations and dilemmas regarding the potential cancelation of the 2016 Olympic Games, had some athletes and fans concerned about the games. Eventually, the Olympic Games were carried out and there is no clear information on the number of infections among athletes or spectators that happened because of the exposure. Transparency and data is the best strategy to avoid public concerns and disbelief. Open communication about Zika cases, geographic regions affected and strategies for vector control preserved public trust in spite of controversies regarding the specific strategies for vector control (local vs aerial spraying). Puerto Rico had public manifestations against the PR Government and the CDC plan to implement aerial spraying with Naled. Even though information regarding the safety of the product and the EPA guidelines, communities and environmental activists protested with marches, demonstrations and media coverage. Eventually, the plan was cancelled. This situation could have been dealt with less tension if all parties had participated in conversations, if activists had been included in the planning and consensus might have been reached. In contrast Miami implemented Naled aerial spraying without significant opposition from communities. Two different approaches and community responses. We do not have data on the efficacy or consequences in those jurisdictions. ${ }^{15-16}$

\section{Overlap between HIV and zika}

What can we learn of the three decades of awareness campaigns, community involvement, clinical, behavior and prevention research, activism and policy-making related to HIV/AIDS? How can the lessons and experience of dealing with the HIV epidemic can help us deal with the ongoing Zika epidemic? Is there a roadmap that we can follow? Our position is that we can and we should. We should have learned from our experience with HIV and use activists and communities' participation to enhance discussions, to disseminate accurate information and to debunk myths. The need to be honest and open with communication is evident. All options need to be considered, including strategies for vector control, expanded testing, awareness and action campaigns.

The comprehensive plan that was developed needs input from communities and other experts. We already have a roadmap if we follow the effective actions we took during the HIV epidemic. Once the initial plan and strategies are in place, the outcomes need to be evaluated and the plan revisited and amended according to experience. Plans need to be as fluid as the epidemic. Because there are no vaccines and no treatments, we need to consider Fast Track mechanisms such as were established by the FDA to allow for the development of potentially life-saving drugs for diseases that have no cure or treatment and have severe outcomes. Because this is a vector-related viral infection, fast track planning and implementation of vector control strategies need to be studied and considered. Zika is transmitted by Aedes species which can transmit dengue, Chikungunya, yellow fever and other viral diseases. Maybe this is the right time to plan for international massive strategies for vector control, which would control and hopefully eliminate many of these tropical diseases.

\section{Recommendations for zilka prevention}

The CDC recommends availability and enhanced testing of symptomatic pregnant women in areas endemic for the mosquito vector and viral infection. Zika virus is primarily transmitted by Aedes aegypti mosquitoes, which are found throughout much of the region of the Americas, including parts of the United States. These mosquitoes can also transmit Dengue and Chikungunya viruses. ${ }^{17}$ According to a CDC report, the Zika virus outbreak continues to spread with ongoing Zika virus transmission in U.S. and its territories both locally acquired and travel-associated. ${ }^{18}$

The ACOG recommendations for preventing Zika include considerations of the following: travel restrictions, avoid mosquito bites including the use of EPA-approved repellents, wearing long sleeves to protect skin, staying in air-conditioned or screened-in areas and the use of condoms during sex or abstaining from sex for the duration of the pregnancy if sex partner has traveled to or lives in an area with active Zika virus transmission.

Essentially the same recommendations were endorsed by multiple groups and the message to pregnant women was: if your partner has travelled to Zika affected areas and you do not live on those geographic areas condoms should be used for at least six months. If you live in a Zika geographic area you should prevent exposure to both mosquito vector and sexual transmission. These recommendations place high burden on individual behavior while generalized community strategies for vector control are in development or have not been implemented.

Some of the comments from the pregnant women with Zika related to the condom use recommendations are: "If I had used the condom that you are giving me today, I would not have been here"; "It might be difficult for me to request the condom use from my partner".

Pregnant women also felt stigmatized and afraid to disclose their status as infected with Zika. They refused to talk with media and some expressed concerns of being blamed for getting the Zika infection. "People might think that I don't care and got this on purpose".

The Zika epidemic requires involvement of multiple sectors with diverse interests and levels of expertise. In order to engage those who can contribute health authorities need to establish a strategic plan. At 
present most local jurisdictions in the US rely on government agencies and collaboration with such partners.

The World Health Organization (WHO) has a framework for strategic planning that includes 4 elements: Detection, Prevention, Care/support and Research. Their first recommended step is to define and prioritize research into Zika virus disease by convening experts and partners followed by to enhance surveillance of Zika virus and potential complications; strengthen capacity in risk communication to engage communities to better understand risks associated with Zika virus. To support health authorities to implement vector control strategies aimed at reducing Aedes mosquito populations and finally to prepare recommendations for the clinical care and follow-up of people with complications related to Zika virus infection, in collaboration with experts and other health agencies. The World Health Organization (WHO) is supporting countries to control Zika virus disease by taking actions outlined in the "Zika Strategic Response Framework". ${ }^{19}$

\section{Lessons from the HIVIAIDS epidemic in the US}

All of these strategies are needed and are vital to control the Zika epidemic. But the consequences of not engaging communities and activists from the beginning will impact the most vulnerable: pregnant women and their infants. We are not listening to the voices of pregnant women in endemic/epidemic areas, we are not including environmental activists and patient advocates in the conversations early on. The lessons of how many countries were able to curtail the growth of the HIV/AIDS epidemic are all around us. One example from the USA is the Ryan White Comprehensive AIDS Resources Emergency Act (Ryan White CARE Act, Pub. L. 101-381, 104 Stat. 576, enacted August 18, 1990). This United States Congress Act is the largest federally funded program in the United States for people living with HIV/AIDS. ${ }^{20}$

The Ryan White Care Program had a strong Community Involvement since the beginning. Consumer representation on planning bodies assure that services reflect the needs of clients and that the way services are offered help them. Consumer Advisory Boards $(\mathrm{CAB})$ were created by grantees and providers to help develop programs that mitigate the impact of stigma and respond to the affected communities. Patients/consumers worked as professionals in the planning, administration, and delivery of HIV/AIDS care services

Some strategic planning activities have relied on expert advice and have neglected community involvement from the beginning. It takes more effort to include opposing views and positions into the conversation and to establish the response to the epidemic. But in the end, the more voices and diversity of opinions, the more options will be considered and maybe a better response or easier acceptance of communities in the prevention and control activities. In the following section, we are going to describe some specific responses to the HIV or the Zika epidemic and the parallels we identify on both issues. We are now describing some of the recommendations that have been published by diverse organizations related to the Zika epidemic and to the HIV/AIDS epidemic.

\section{Recommendations for HIV prevention and treatment}

Throughout the many decades of the AIDS epidemic recommendations have changed according to the development of prevention and treatment options and to the evidence of their efficacy.
Initially most recommendations focused on condom use, testing and reducing behavior risk such as reducing numbers of sexual partners, engaging in safer injection use practices and encouraging monogamy. The initial messages for AIDS prevention focused on the "ABC of AIDS: Abstinence, Be-faithful and use Condoms". These messages were included in a variety of forms during public education and awareness campaigns and are still present in WHO and PEPFAR strategies. ${ }^{12-14}$

Over the past 25 years, HIV/AIDS has become part of the landscape of the contemporary world. ${ }^{21}$ Since then, many people have worked together to end this epidemic. Mayor efforts have been made in order to develop different strategies for prevention. Consistent condom use has long been advocated as the most effective prevention practice in decreasing HIV risk inherent in heterosexual intercourse. ${ }^{22}$ Efficacy trials of behavioral interventions that promote condom use have shown promise; however, improving condom use in real-world settings over an extended time remains a challenge, particularly for the highest risk groups. ${ }^{23}$ Research has shown that condom use is a multifaceted issue, particularly in sexual relationships involving power differentials and perceived monogamy. For example a study conducted by Bonacquisti \& Geller ${ }^{24}$ showed that when women's preference to use condoms was compared to their report of actual condom-use behavior, significant discrepancies emerged, indicating that women often are not in control of this aspect of their sexual behavior. ${ }^{24}$ Moreover, another study showed that among 1,252 women enrolled, $78.4 \%$ were married (of whom $15.1 \%$ were in polygamous unions), $1.4 \%$ had a known HIV-infected partner, and 33.6\% partner of unknown HIV status. At enrollment, 58.5\% reported sex in past month of which the majority (94.3\%) was unprotected. ${ }^{25}$ Although many structural barriers to condom use exist, numerous studies demonstrate that HIV risk reduction through condom use is strongly associated with relationship dynamics between sexual partners. ${ }^{26-30} \mathrm{As}$ we know, ZIKA is a sexual transmitted infection as well as HIV. We have learned that condom use in high-risk groups (such as pregnant women) is not as effective as we expected. We have an opportunity to implement other strategies that actually work in order to reduce the spread of ZIKA.

Pregnancy prevention of Mother-to-child transmission (MTCT) was demonstrated with landmark study on the use of Zidovudine during pregnancy, labor and to the infant. The PACTG-076 study provided the evidence of what we now call pre-exposure prophylaxis (PrEP) by having a maternal antiviral use during pregnancy and post-exposure prophylaxis (PEP) by administering the same drug to the infants for 6 weeks. The efficacy of these strategies increased remarkably with the use of more potent antiviral drugs known as Highly Active AntiRetroviral Therapy (HAART). The implementation of universal prenatal HIV screening, HAART, elective cesarean delivery, and use of infant formula instead of breastfeeding, the rate of MTCT decreased to $2 \%$ or less in the United States and Europe. ${ }^{31-32}$

Guidelines now recommend HAART for every person identified as living with HIV ${ }^{33-34}$ and PrEP for those at risk (ref 5-6). In essence, biomedical advances made the treatment of HIV infection also a preventive strategy since people on effective treatment reduce significantly their transmission risk (to almost zero). In addition some of the HIV antiviral drugs were demonstrated to be effective in reducing the risk of acquisition of HIV which led to the new and current recommendations for the use of PrEP. ${ }^{35-36}$ For HIV infection we have effective strategies to reduce perinatal (MTCT) transmission as well as the risk of acquisition. Better care means longer life expectancy and better quality of life. 
In spite of an ongoing epidemic or pandemic, growing reports of Zika-related congenital anomalies and adverse pregnancy outcomes, the WHO decided on a surprising and unanticipated statement to declare the end of the Public Health Emergency of International Concern (PHEIC) ${ }^{37}$ The response to the WHO decision has been of concern with the potential public perception that the Zika epidemic is over and the potential decrease in intensity of funding and activities to curtail the epidemic. Since the most affected populations are pregnant women and their infants we question the decision and agree with the concerns with the outcomes of this decision.

\section{Recommendations}

We propose the establishment of a commission or work group for fast action with national and international perspective. We know that strategic plans have been developed by international agencies like WHO and US agencies including the CDC and the NIH. This group should have representation of not only government agencies responsible for the epidemic response, but also community representatives, environmental activists, pregnant women, representatives of the ObGyn physicians, pediatricians and scientists. We propose a Fast track process for the approval of strategies that have been demonstrated effective in vector control and have not been implemented. Examples are the AGO traps and the Sterile Insect technique strategies (SIT). ${ }^{38-40}$

The implementation and distribution of the strategies should be accelerated and we should not wait for additional studies as proposed with the AGO traps in Puerto Rico. The current reporting system for the Zika tests should be improved substantially.

The infrastructure for diagnostic tests in the US is currently limited to the local Health Departments and CDC-sponsored laboratories and it should be expanded to other experienced laboratories and centers with experience in clinical research and specialized testing. We acknowledge that for this to happen tests need to be developed, tested and demonstrated effective for generalized use.

Positive and negative results need to be provided in a shorter time-around so that pregnant patients have real options and quicker evaluations during the pregnancy. Patients should have access to their results. All of the laboratories and imaging studies centers in the US and its territories provide the results directly to the patients. Testing frequency during pregnancy should be increased to include the third trimester and potentially the delivery time if the woman has not been tested. The purpose of this expanded pregnancy testing in affected areas is to identify at-risk or Zika-exposed infants.

\section{Conclusion}

Parallels between the Zika and HIV/AIDS epidemics particularly in relation to pregnancy can be established. Lessons and successes from the HIV/AIDS epidemic control efforts can guide us towards comprehensive approaches to improve the health of women and infants at risk for Zika.

\section{Acknowledgments}

None.

\section{Conflicts of interest}

None.

\section{References}

1. WHO. World Health Organization, WHO statement on the first meeting of the International Health Regulations (2005) (IHR 2005) Emergency Committee on Zika virus and observed increase in neurological disorders and neonatal malformations. 2016.
2. Farlex Inc. AIDS embryopathy. 2016.

3. Brasil P, Pereira JP Jr, Moreira ME, et al. Zika Virus Infection in Pregnant Women in Rio de Janeiro - Preliminary Report. $N$ Engl J Med [Epub ahead of print]. 2016.

4. The Henry J. Kaiser Family Foundation. WHO/PAHO Release Updated Zika Strategic Response Plan, Call For \$121.9M In Funding. 2016.

5. World Health Organization. ZIKA Strategic Response Plan Quarterly updated July-September 2016. 2016;P.1-29.

6. World Health Organization. Zika virus outbreak global response. 2016.

7. The American Congress of Obstetricians and Gynecologists. Practice Advisory on Zika Virus. 2016.

8. D McNeil Jr. First Baby in Puerto Rico With Zika-RelatedMicrocephaly Born, The New York Times. 2016.

9. L Sun. WHO no longer considers Zika a global health emergency, World Health Organization. 2016.

10. J Reitman. Zika: una epidemia que se expande, Rolling Stones. 2016.

11. CBS 60 minutes. Morning Rounds: Zika's effects, sleep guidelines and more, CBS, USA. 2016.

12. AIDS Centre. ABC of HIV Prevention, 2002-2010.

13. Office of U.S. Global AIDS Coordinator and the Bureau of Public Affairs, The United States President's Emergency Plan for AIDS relief. Defining the ABC Approach. 2016.

14. Avert. HIV Prevention Programmes Overview. 2016.

15. Caribbean Business. Anti-Zika Fumigation Plans in Puerto Rico Cause Outrage. 2016.

16. Reuters. Florida to Start Aerial Spraying to Combat Zika, Fortune. 2016

17. Centers for Disease Control and Prevention. Transmission \& Risks. 2016.

18. Centers for Disease Control and Prevention. Case count in the US. 2016.

19. World Health Organization. Zika Virus Fact Sheet. 2016.

20. Health Resources \& Services Administration. Ryan White HIV/AIDS Program Legislation. 2016.

21. Merson MH, O'Malley J, Serwadda D, et al. The history and challenge of HIV prevention. Lancet. 2008;372(9637):475-488.

22. Weller S, Davis K. Condom effectiveness in reducing heterosexual HIV transmission (review). Cochrane Database Syst. 2002;Rev4:1-22.

23. Noar SM. Behavioral interventions to reduce HIV-related sexual risk behavior: review and synthesis of meta-analytic evidence. AIDS Behav. 2008;12(3):335-353.

24. Bonacquisti A, Geller PA. Condom-use intentions and the influence of partner-related barriers among women at risk for HIV. J Clin Nurs. 2013;22(23-24):3328-3336.

25. Kinuthia J, Richardson BA, Drake AL, et al. Sexual Behavior and Vaginal Practices during Pregnancy and Postpartum: Implications for HIV Prevention Strategies. J Acquir Immune Defic Syndr [Epub ahead of print]. 2016.

26. El-Bassel N, Gilbert L, Rajah V, et al. Fear and violence: raising the HIV stakes. AIDS Educ Prev. 2000;12(2):154-170.

27. A Gielen, K McDonnell, P O'Campo. "Intimate partner violence, HIV status, and sexual risk reduction. AIDS and Behavior. 2002;6(2):107116.

28. Dunkle KL, Jewkes RK, Brown HC, et al. Gender-based violence, relationship power, and risk of HIV infection in women attending antenatal clinics in South Africa. Lancet. 2004;363(9419):1415-1421. 
29. Harvey SM, Beckman LJ, Gerend MA, et al. A conceptual model of women's condom use intentions: integrating intrapersonal and relationship factors. AIDS Care. 2006;18(7):698-709.

30. Seth P, Raiford JL, Robinson LS, et al. Intimate partner violence and other partner-related factors: correlates of sexually transmissible infections and risky sexual behaviors among young adult African American women. Sex Health. 2010;7(1):25-30.

31. Townsend CL, Byrne L, Cortina-Borja M, et al. Earlier initiation of ART and further decline in mother-to-child HIV transmission rates, 2000-2011. AIDS. 2014;28(7):1049-1057.

32. Centers for Disease Control and Prevention. Enhanced perinatal surveillance-15 areas, 2005-2008. HIV Surveillance Supplemental Report. 2011;16(2):1-32.

33. Department of Health and Human Services, Panel on Antiretroviral Guidelines for Adults and Adolescents, Guidelines for the use of antiretroviral agents in HIV-1-infected adults and adolescents. 2016.

34. World Health Organization. Consolidated guidelines on the use of antiretroviral drugs for treating and preventing HIV infection Recommendations for a public health approach - Second edition. 2016
35. Centers for Disease Control and Prevention. Preexposure Prophylaxis for the Prevention of HIV Infection in the United States - 2014 Clinical Practice Guideline. 2014;p.1-67.

36. World Health Organization. Guidance on oral pre-exposure prophylaxis (PrEP) for serodiscordant couples, men and transgender women who have sex with men at high risk of HIV. 2012.

37. World Health Organization. Fifth meeting of the Emergency Committee under the International Health Regulations (2005) regarding microcephaly, other neurological disorders and Zika virus. 2016.

38. Barrera R, Amador M, Acevedo V, et al. Use of the CDC autocidal gravid ovitrap to control and prevent outbreaks of Aedes aegypti (Diptera: Culicidae). J Med Entomol. 2014;51(1):145-154.

39. Centers for Disease Control and Prevention. Reduced Incidence of Chikungunya Virus Infection in Communities with Ongoing Aedes Aegypti Mosquito Trap Intervention Studies - Salinas and Guayama, Puerto Rico, November 2015-February 2016.

40. H Araújo, D Carvalho, R Ioshino, et al. "Aedes aegyptiControl Strategies in Brazil: Incorporation of New Technologies to Overcome the Persistence of Dengue Epidemics. Insects. 2015;6(2):576-594. 